\title{
TRIPLE-DIFFUSIVE CONVECTION IN WALTERS' (MODEL B') FLUID WITH VARYING GRAVITY FIELD SATURATING A POROUS MEDIUM
}

\author{
S.K. KANGO \\ Department of Mathematics, Government College, \\ Haripur (Manali) - 175 136, Himachal Pradesh, India. E-mail: skkango72@gmail.com \\ G.C. RANA \\ Department of Mathematics, NSCBM Government PG College, Hamirpur, India.
}

RAMESH CHAND

Department of Mathematics, Government College, Dhaliara (HP), India.

\begin{abstract}
The Triple-Diffusive convection in Walters' (Model B') fluid with varying gravity field is considered in the presence of uniform vertical magnetic field in porous medium. For the case of stationary convection, the magnetic field, varying gravity field and the stable solute gradients have stabilizing effects whereas the medium permeability has destabilizing (or stabilizing) effect on the system under certain conditions. A linear stability analysis theory and normal mode analysis method have been carried out to study the onset convection. The kinematic viscoelasticity has no effect on the stationary convection. The solute gradients, magnetic field, varying gravity field, porosity and kinematic viscoelasticity introduce oscillatory modes in the system, which were non-existent in their absence. The sufficient conditions for the non-existence of overstability are also obtained. The results are also shown graphically.
\end{abstract}

\section{LIST OF SYMBOLS}

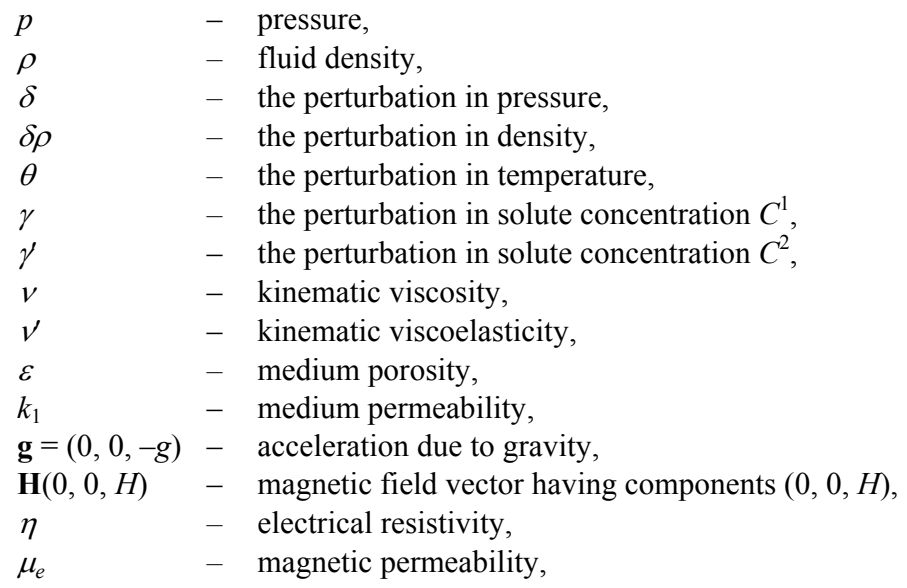




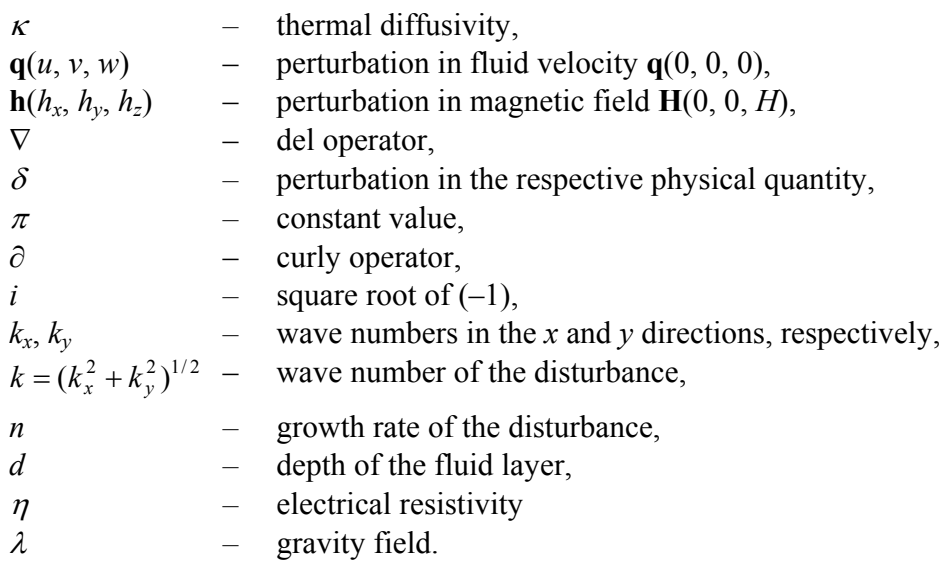

\section{INTRODUCTION}

The instability of the plane interface separating two Newtonian fluids when one is superposed over the other, under varying assumptions of hydrodynamics and hydromagnetics, has been studied by several researchers and a comprehensive account of these investigations has been given by Chandrasekhar [1]. The problem of thermohaline convection in a layer of fluid heated from below and subjected to a stable salinity gradient has been considered by Veronis [11].

With the growing importance of non-Newtonian fluids in geophysical fluid dynamics, chemical technology and petroleum industry, the investigations on such fluids are desirable. The Walters' (Model B') fluid [12] is one such fluid.

The flow through porous medium has been of considerable interest in recent years, particularly in geophysical fluid dynamics. A porous medium is a solid with holes in it, and is characterized by the manner in which the holes are imbedded, how they are interconnected and the description of their location, shape and interconnection. However, the flow of a fluid through a homogeneous and isotropic porous medium is governed by Darcy's law which states that the usual viscous term in the equations of Walters' (Model B') fluid motion is replaced by the resistance term $\left[-\frac{1}{k_{1}}\left(\mu-\mu^{\prime} \frac{\partial}{\partial t}\right)\right] q$, where $\mu^{\prime}$ and $\mu^{\prime}$ are the viscosity and viscoelasticity of the incompressible Walters' (Model B') fluid, $k_{1}$ is the medium permeability, and $q$ is the Darcian (filter) velocity of the fluid.

In the physical world, the investigation of flow of Rivlin-Ericksen fluid through porous medium has become an important topic due to the recovery of crude oil from the pores of reservoir rocks. Flows in porous region are a creeping flow. When a fluid permeates a porous material, the actual path of the individual particles cannot be fol- 
lowed analytically. When the density of a stratified layer of a single-component fluid decreases upwards, the configuration is stable. This is not necessarily so far a fluid consisting of two or more components which can diffuse relative to each other. The reason lies in the fact that the diffusivity of heat is usually much greater than the diffusivity of a solute. A displaced particle of fluid thus, loses any excess heat more rapidly than any excess solute. The resulting buoyancy force may tend to increase the displacement of the particle from its original position and thus cause instability.

The idealization of uniform gravity field can be hardly justified in the presence of large scale convection phenomenon occurring in atmosphere, the ocean or the mantle of the earth. Pradhan et al [6] studied the thermal instability of the fluid layer under variable gravitational field.

A comprehensive review of the literature concerning thermosolutal convection in a fluid-saturated porous medium may be found in the book written by Nield and Bejan [3]. The thermosolutal convection in Rivlin-Ericksen fluid in the presence of Hall currents in porous medium in hydromagnetics has been studied by Kango and Singh [2]. Sharma R.C., Sunil and Suresh Chand [7] have studied thermosolutal convection in Walters' (Model B') fluid in porous medium in hydrodynamics whereas the effect of rotation on triple-diffusive convection in Walters' (Model B') fluid in porous medium has been investigated by Suresh Chand [10]. Oldenburg and Pruess [4] have developed a model for convection in a Darcy's porous medium, where the mechanism involves temperature, $\mathrm{NaCl}, \mathrm{CaCl}_{2}$ and $\mathrm{KCl}$.

In the standard Benard problem, the instability is driven by a density difference caused by a temperature difference between the upper and lower planes bounding the fluid. If the fluid additionally has salt dissolved in it, then there are potentially two destabilizing sources for the density difference, the temperature field and the salt field. The solution behaviour in the double-diffusive convection problem is more interesting than that of the single component situation in so much as new instability phenomena may occur, which is not present in the classical Benard problem. When temperature and two or more agencies, or two different salts, are present the physical and mathematical situation becomes increasingly richer. Very interesting results in triplydiffusive convection have been obtained by Pearlstein et al. [5]. They demonstrate that for triple diffusive convection linear instability can occur in discrete sections of the Rayleigh number domain with the fluid being linearly stable in a region in between the linear instability ones. This is because for certain parameters the neutral curve has a finite isolated oscillatory instability curve lying below the usual unbounded stationary convection one. Straughan and Walker [9] derive the equation for non-Boussinesq convection in a multi-component fluid and investigate the situation analogous to that of Pearlstein et al., but allowing for a density non-linear in a temperature field. In reality the density of a fluid is never a linear function of temperature, and so the work of Straughan and Walker applies to the general situation where the equation of state is one of the density quadric in temperature. This is important, since they find that de- 
parture from the linear Boussinesq equation of state changes the perfect symmetry of the heart shaped neutral curve of Pearlstein et al.

Keeping in view the recent increase in the number of non iso-thermal situations and the importance of non-Newtonian fluid applications in the industries, our interest, in the present paper is to study the triple-diffusive convection in Walters' (Model B') fluid with varying gravity field saturating a porous medium.

\section{MATHEMATICAL FORMULATION OF THE PROBLEM}

Consider an infinite, horizontal, incompressible layer of thickness $d$ of an electrically non-conducting Walters' (Model B') fluid heated and salted from below. The temperature $T$ and solute concentrations $C^{1}$ and $C^{2}$ at the bottom and top surfaces $z=0, d$ are $T_{0}$ and $T_{1} ; C_{0}^{1}, C_{1}^{1}$ and $C_{0}^{2}, C_{1}^{2}$, respectively, and a uniform temperature gradient $\beta(=|\mathrm{d} T / \mathrm{d} z|)$ and uniform solute gradients $\beta^{\prime}\left(=\left|\mathrm{d} C^{1} / \mathrm{d} z\right|\right)$ and $\beta^{\prime \prime}\left(=\left|\mathrm{d} C^{2} / \mathrm{d} z\right|\right)$ are maintained. A uniform magnetic field $\mathbf{H}=(0,0, H)$ and the gravity field $\mathbf{g}=(0,0,-g)$, where $\mathbf{g}=g_{0} \lambda,\left(g_{0}>0\right)$ is the value of $g$ at $z=0$ and $\lambda$ can be $+v e$ or $-v e$ according to whether gravity increases or decreases upwards from its value $g_{0}$; pervades the system.

The equations expressing the conservation of momentum, mass, temperature, solute concentrations and equation of Walters' (Model B') fluid are

$$
\begin{gathered}
\frac{1}{\varepsilon}\left[\frac{\partial \mathbf{q}}{\partial t}+\frac{1}{\varepsilon}(\mathbf{q} \cdot \nabla) \mathbf{q}\right]=-\left(\frac{1}{\rho_{0}}\right) \nabla p+\mathbf{g}\left(1+\frac{\delta \rho}{\rho_{0}}\right)-\frac{1}{k}\left(v-v^{\prime} \frac{\partial}{\partial t}\right) \mathbf{q}+\frac{\mu_{e}}{4 \pi \rho_{0}}(\nabla \times \mathbf{H}) \times \mathbf{H}, \\
\nabla \cdot \mathbf{q}=0 \\
E=\frac{\partial T}{\partial t}+(q . \nabla) T=\kappa \nabla^{2} T \\
E^{\prime} \frac{\partial C^{1}}{\partial t}+(q . \nabla) C^{1}=\kappa^{\prime} \nabla^{2} C^{1} \\
E^{\prime \prime} \frac{\partial C^{2}}{\partial t}+(q . \nabla) C^{2}=\kappa^{\prime \prime} \nabla^{2} C^{2}
\end{gathered}
$$

The density equation of state is

$$
\rho=\rho_{0}\left[1-\alpha\left(T_{1}-T_{0}\right)+\alpha^{\prime}\left(C_{1}^{1}-C_{0}^{1}\right)+\alpha^{\prime \prime}\left(C_{1}^{2}-C_{0}^{2}\right)\right],
$$

where $\rho, \rho_{0}, \mathbf{q}, t, \mathbf{g}, v, v^{\prime}, \kappa, \kappa^{\prime}, \kappa^{\prime \prime}, \alpha, \alpha^{\prime}, \alpha^{\prime \prime}$ are the fluid density, reference density, velocity, time, gravitational acceleration, the kinematic viscosity, the kinematic viscoelasticity, the thermal diffusivity, the solute diffusivities $\kappa^{\prime}$ and $\kappa^{\prime \prime}$, thermal and 
solvent coefficients of expansion $\alpha^{\prime}$ and $\alpha^{\prime \prime}$, respectively. $T_{a}$ is the average temperature given by $T_{a}=\left(T_{0}+T_{1}\right) / 2$ where $T_{0}$ and $T_{1}$ are the constant average temperatures of the lower and upper surfaces of the layer and $C_{a}^{1}, C_{a}^{2}$ are the average concentrations given by $C_{a}^{1}=\left(C_{0}^{1}+C_{1}^{1}\right) / 2$ and $C_{a}^{2}=\left(C_{0}^{2}+C_{1}^{2}\right) / 2$, where $C_{0}^{1}, C_{1}^{1}$ and $C_{0}^{2}, C_{1}^{2}$ are the constant average concentrations of the lower and upper surfaces of the layer. In writing equation (2), we also use the Boussinesq approximation by allowing the density to change only in the gravitational body force term. When the permeability of the porous material is low, then the inertial force becomes relatively insignificant as compared with the viscous drag when flow is considered. And as we know $\frac{1}{\varepsilon}(\mathbf{q} . \nabla) \mathbf{q}$ term is generally small, so it seems best to drop it in numerical work.

Maxwell's equations yield

$$
\begin{gathered}
\varepsilon \frac{\partial \mathbf{H}}{\partial t}=(\mathbf{H} . \nabla) \mathbf{q}+\varepsilon \eta \nabla^{2} \mathbf{H}, \\
\nabla . \mathbf{H}=0 .
\end{gathered}
$$

Here, $E=\varepsilon+(1-\varepsilon) \frac{\rho_{s} C_{s}}{\rho_{0} C_{i}}$ is a constant, $E^{\prime}$ and $E^{\prime \prime}$ are analogous to $E$ but corresponding to solute rather than heat. $\rho_{s}, C_{s}$ and $\rho_{0}, C_{i}$ stand for density and heat capacity of solid (porous) and fluid, respectively.

The steady state solution is

$$
\begin{gathered}
\mathbf{q}=(0,0,0), \quad T=-\beta z+T_{a}, \quad C^{1}=-\beta^{\prime} z+C_{a}^{1}, \quad C^{2}=-\beta^{\prime \prime} z+C_{a}^{2}, \quad \beta=\left(T_{0}-T_{1}\right) / d \\
\beta^{\prime}=\left(C_{1}^{1}-C_{0}^{1}\right) / d, \quad \beta^{\prime \prime}=\left(C_{1}^{2}-C_{0}^{2}\right) / d, \quad \rho=\rho_{0}\left(1+\alpha \beta z-\alpha^{\prime} \beta^{\prime} z-\alpha^{\prime \prime} \beta^{\prime \prime} z\right) .
\end{gathered}
$$

Here we use the linearized stability theory and the normal mode method. Consider a small perturbation on the steady state solution and let $\delta p, \delta \rho, \theta, \gamma, \gamma^{\prime}, \mathbf{h}\left(h_{x}, h_{y}, h_{z}\right)$ and $\mathbf{q}(u, v, w)$ denote, respectively, the perturbation in pressure $p$, density $\rho$, temperature $\mathrm{T}$, solute concentrations $C^{1}, C^{2}$, the magnetic field $\mathbf{H}(0,0, H)$ and denote $\mathbf{q}(0,0,0)$. The change in density $\delta \rho$, caused mainly by the perturbations $\theta$ in temperature and $\gamma$, $\gamma^{\prime}$ in concentrations, is given by

$$
\delta \rho=-\rho_{0}\left(\alpha \theta-\alpha^{\prime} \gamma-\alpha^{\prime \prime} \gamma^{\prime}\right) .
$$

Then the linearized perturbation equations become

$$
\frac{1}{\varepsilon} \frac{\partial \mathbf{q}}{\partial t}=-\left(\frac{1}{\rho_{0}}\right) \nabla \delta p-g_{0} \lambda\left(\alpha \theta-\alpha^{\prime} \gamma-\alpha^{\prime \prime} \gamma^{\prime}\right)-\frac{1}{k}\left(v-v^{\prime} \frac{\partial}{\partial t}\right) \mathbf{q}+\frac{\mu_{e}}{4 \pi \rho_{0}}(\nabla \times \mathbf{H}) \times \mathbf{H},
$$




$$
\begin{gathered}
\nabla \cdot \mathbf{q}=0 \\
E=\frac{\partial \theta}{\partial t}=\beta w+\kappa \nabla^{2} \theta, \\
E^{\prime}=\frac{\partial \gamma}{\partial t}=\beta^{\prime} w+\kappa^{\prime} \nabla^{2} \gamma, \\
E^{\prime \prime}=\frac{\partial \gamma^{\prime}}{\partial t}=\beta^{\prime \prime} w+\kappa^{\prime \prime} \nabla^{2} \gamma^{\prime}, \\
\varepsilon \frac{\partial h}{\partial t}=(\mathbf{H} \cdot \nabla)+\varepsilon \eta \nabla^{2} \mathbf{h}, \\
\nabla . \mathbf{h}=0 .
\end{gathered}
$$

\section{DISPERSION RELATION}

Analyzing the disturbances into normal modes, we assume that the perturbation quantities are of the form

$$
\left[w, \theta, \gamma, \gamma^{\prime}, h_{z}\right]=[W(z), \Theta(z), K(z), \Gamma(z), \Psi(z)] \exp i\left(i k_{x} x+i k_{y} y+n t\right),
$$

where $k_{x}, k_{y}$ are the wave numbers along the $x$ and $y$ directions, respectively, and $k=\sqrt{k_{x}^{2}+k_{y}^{2}}$ is the resultant wave number and $n$ is, in general, a complex constant.

Equations (11)-(17), using (18), in non-dimensional form become

$$
\begin{gathered}
{\left[\frac{\sigma}{\varepsilon}+\frac{1}{P_{l}}(1-F \sigma)\right]\left(D^{2}-a^{2}\right) W+\frac{g_{0} \lambda a^{2} d^{2}}{v} g_{0} \lambda\left(\alpha \Theta-\alpha^{\prime} \Gamma-\alpha^{\prime \prime} \Psi\right)} \\
-\frac{\mu_{e} H}{4 \pi \rho_{0} v}\left(D^{2}-a^{2}\right) D K=0 \\
\left(D^{2}-a^{2}-p_{2} \sigma\right) K=-\left(\frac{H d}{\varepsilon \eta}\right) D W \\
\left(D^{2}-a^{2}-E p_{1} \sigma\right) \Theta=-\left(\frac{\beta d^{2}}{\kappa}\right) W
\end{gathered}
$$




$$
\begin{gathered}
\left(D^{2}-a^{2}-E^{\prime} q_{1} \sigma\right) \Gamma=-\left(\frac{\beta^{\prime} d^{2}}{\kappa^{\prime}}\right) W, \\
\left(D^{2}-a^{2}-E^{\prime \prime} q_{2} \sigma\right) \Psi=-\left(\frac{\beta^{\prime \prime} d^{2}}{\kappa^{\prime \prime}}\right) W .
\end{gathered}
$$

Consider the case where both the boundaries are free as well as perfect conductors of heat and concentrations. The case of two free boundaries is a little artificial but it enables us to find analytical solutions and to make some qualitative conclusions. The appropriate boundary conditions w.r.t. which equations (19)-(23) must be solved (Chandrasekhar [1]).

$$
W=D^{2} W=0, \Theta=\Gamma=\Psi=0, \text { at } z=0 \text { and } z=1,
$$

$K=0$ on the perfectly conducting boundaries and $h_{x}, h_{y}, h_{z}$ are continuous.

The case of two free boundaries, though little artificial, is the most appropriate for stellar atmospheres (Spiegel [8]). Using the above boundary conditions, it can be shown that all the even order derivatives of $W$ must vanish for $z=0$ and $z=1$ and hence the proper solution of $W$ characterizing the lowest mode is

$$
W=W_{0} \sin \pi z,
$$

where $W_{0}$ is a constant.

Eliminating $\Theta, \Gamma, K$ and $\Psi$ between equations (19)-(23) and substituting (25), we obtained the dispersion relation

$$
\begin{aligned}
R_{1}=\frac{1+x}{x \lambda}\left[\frac{i \sigma_{1}}{\varepsilon}\right. & \left.+\frac{1}{P}\left(1-i F \sigma_{1}\right)\right]\left(1+x+i E p_{1} \sigma_{1}\right)+\frac{Q_{1}}{\lambda}\left[\frac{(1+x)\left(1+x+i E p_{1} \sigma_{1}\right)}{x\left(1+x+i p_{2} \sigma_{1}\right)}\right] \\
& +S_{1} \frac{\left(1+x+i E p_{1} \sigma_{1}\right)}{\left(1+x+i E^{\prime} q_{1} \sigma_{1}\right)}+S_{1} \frac{\left(1+x+i E p_{1} \sigma_{1}\right)}{\left(1+x+i E^{\prime \prime} q_{2} \sigma_{1}\right)}
\end{aligned}
$$

where

$$
\begin{gathered}
R_{1}=\frac{g \alpha \beta d^{4}}{v \kappa \pi^{4}}, S_{1}=\frac{g \alpha^{\prime} \beta^{\prime} d^{4}}{v \kappa^{\prime} \pi^{4}}, \quad S_{2}=\frac{g \alpha^{\prime \prime} \beta^{\prime \prime} d^{4}}{v \kappa^{\prime \prime} \pi^{4}}, \quad Q_{1}=\frac{\mu_{e} H^{2} d^{2}}{4 \pi \rho_{0} v \eta \varepsilon \pi^{2}}, \\
x=\frac{a^{2}}{\pi^{2}}, \quad P_{l}=\frac{P}{\pi^{2}} \text { and } i \sigma_{1}=\frac{\sigma}{\pi^{2}} .
\end{gathered}
$$

Equation (26) the required dispersion relation studying the effects of magnetic field, variable gravity field, medium permeability, kinematic viscoelasticity and stable solute gradients on the triple-diffusive convection of Walters' (Model B') fluid in porous medium. 


\section{STATIONARY CONVECTION}

For the case of stationary convection, $\sigma=0$ and equation (26) reduces to

$$
R_{1}=\frac{(1+x)^{2}}{x P \lambda}+Q_{1} \frac{1+x}{x \lambda}+S_{1} \lambda+S_{2} \lambda,
$$

which expresses the modified Rayleigh number $R_{1}$ as a function of the dimensionless wave number $x$ and the parameters $Q_{1}, S_{1}, S_{2}$ and $P$. For stationary convection the parameter $F$ accounting for the kinematic viscoelasticity effect vanishes and thus the Rivlin-Ericksen elastico-viscous fluid behaves like an ordinary Newtonian fluid.

To investigate the effects of stable solute gradients, magnetic field and the medium permeability, we examine the behaviour of $\frac{d R_{1}}{d S_{1}}, \frac{d R_{1}}{d S_{2}}, \frac{d R_{1}}{d Q_{1}}$ and $\frac{d R_{1}}{d P}$.

Equation (27) yields

$$
\frac{d R_{1}}{d S_{1}}=+1, \quad \frac{d R_{1}}{d S_{2}}=+1,
$$

implying that the stable solute gradients have a stabilizing effect on the system,

$$
\frac{d R_{1}}{d Q_{1}}=\frac{1+x}{x \lambda} .
$$

The magnetic field, therefore, has a stabilizing effect on the system when gravity is increasingly upwards $(\lambda>0)$ and destabilizes the system when gravity is decreasing upwards,

$$
\frac{d R_{1}}{d P}=\frac{(1+x)^{2}}{x P \lambda}
$$

which is always negative, showing that medium permeability has a destabilizing effect on the system and for constant varying gravity.

The dispersion relation (27) is analyzed numerically. In Fig. $1, R_{1}$ is plotted against $x$ for $Q_{1}=30, P=1, \lambda=2, S_{2}=100$ and $S_{1}=100,200,300$. The stabilizing role of solute gradient is clear from the increase in the Rayleigh number with increasing parameter value $S_{1}$. In Fig. $2, R_{1}$ is plotted against $x$ for $Q_{1}=30, P=1, \lambda=2, S_{1}=100$ and $S_{2}=100,200,300$. The stabilizing role of solute gradient is clear from the increase in the Rayleigh number with increasing parameter value $S_{2}$. In Fig. $3, R_{1}$ is plotted against $x$ for $P=1, \lambda=2, S_{1}=100$ and $S_{2}=100$ and $Q_{1}=30,60,90$. The stabilizing role of magnetic field is clear from the increase in the Rayleigh number with increasing parameter value $Q_{1}$. In Fig. $4, R_{1}$ is plotted against $x$ for $\mathrm{Q} 1=30, \lambda=2$, $S_{1}=100, S_{2}=100$ and $P=2,4,6$. The destabilizing role of medium permeability is clear from the decrease in the Rayleigh number with increasing parameter value $S_{2}$. 


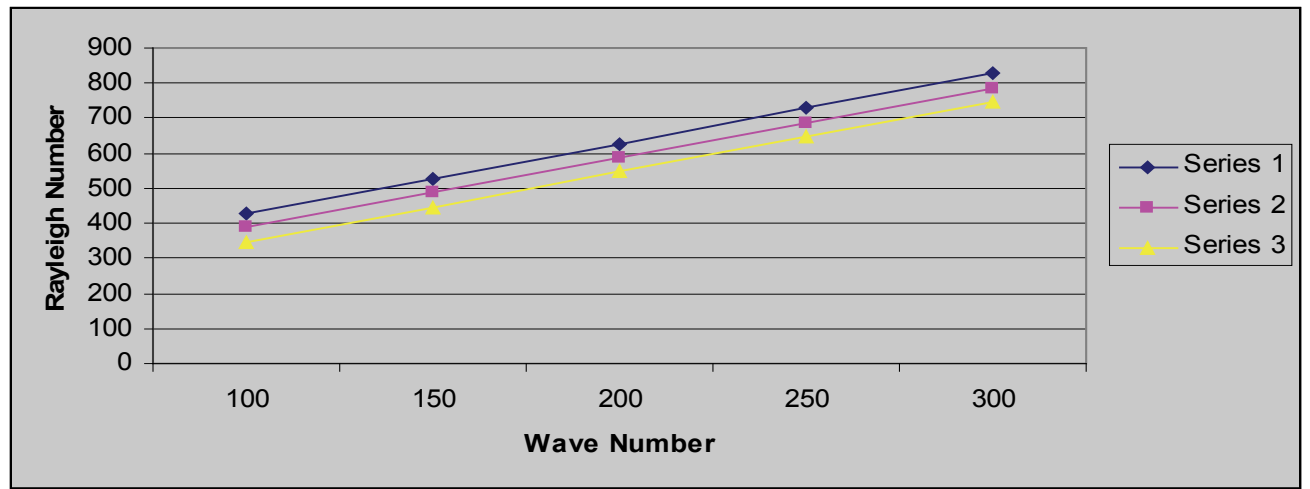

Fig. 1. The stabilizing effect of stable solute gradient $S_{1}$

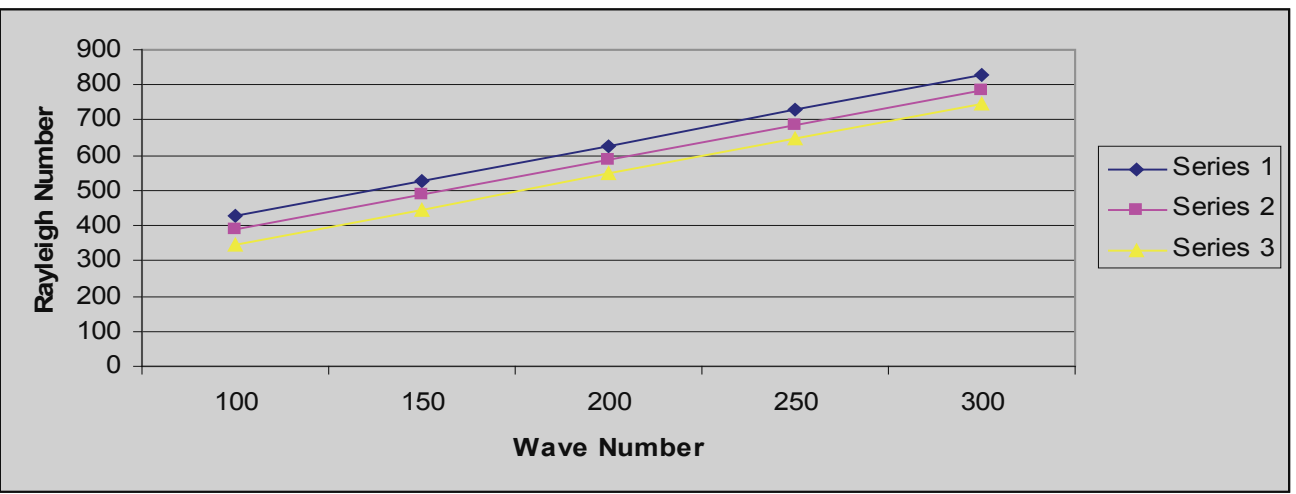

Fig. 2. The stabilizing effect of stable solute gradient $S_{2}$

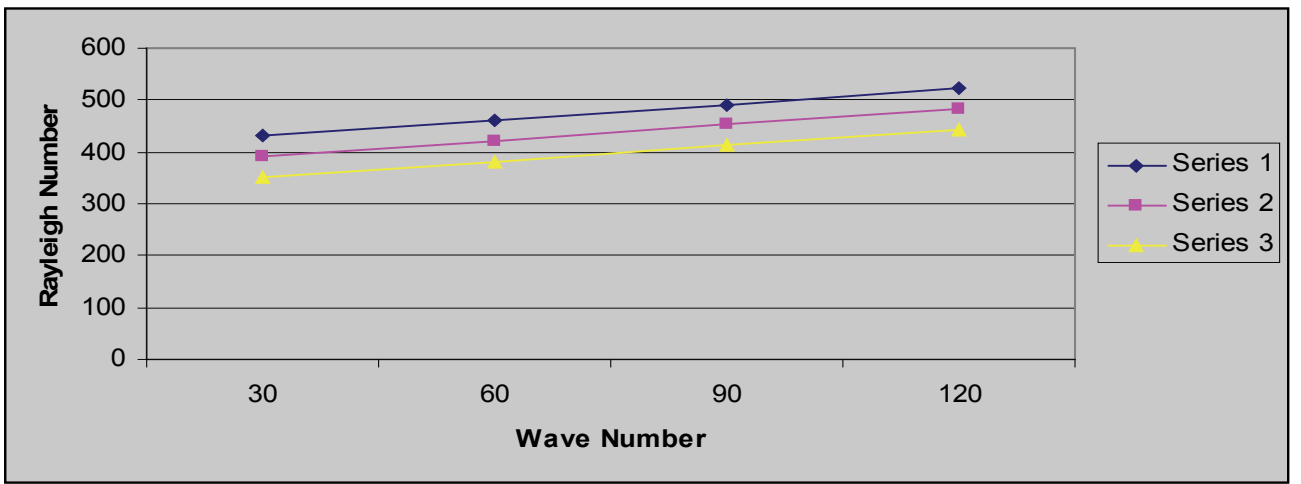

Fig. 3. The stabilizing effect of magnetic field 


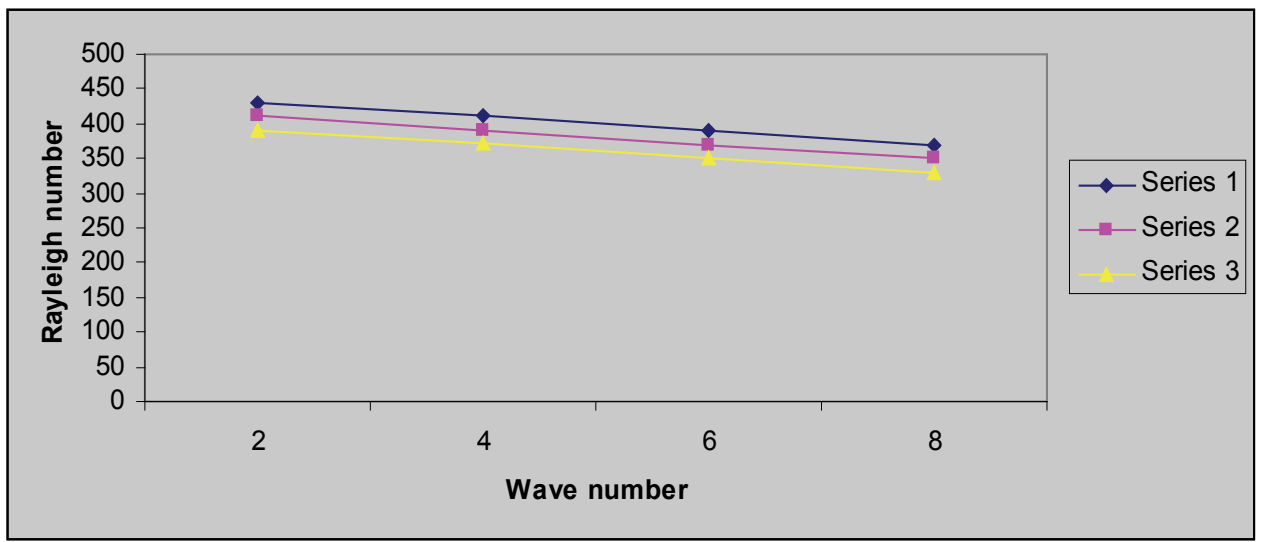

Fig. 4. The destabilizing effect of medium permeability

\section{STABILITY OF THE SYSTEM AND OSCILLATORY MODES}

Here we examine the possibility of oscillatory modes, if any, in the stability problem due to the presence of magnetic field, varying gravity field, kinematic viscoelasticity and stable solute gradients. Multiplying equation (19) by $W^{*}$, the complex conjugate of $W$, integrating over the range of $z$ and making use of equations (23)-(27) together with the boundary conditions (29) and putting $\sigma=\sigma_{r}+i \sigma_{i}$ and equating the real and imaginary parts, we obtain

$$
\begin{gathered}
{\left[\left(\frac{1}{\varepsilon}-\frac{F}{P_{l}}\right) I_{1}-\left(\frac{g_{0} \lambda \alpha \kappa a^{2}}{v \beta}\right) E p_{1} I_{3}+\left(\frac{g_{0} \lambda \alpha^{\prime} \kappa^{\prime} a^{2}}{v \beta^{\prime}}\right) E^{\prime} q_{1} I_{5}+\left(\frac{g_{0} \lambda \alpha^{\prime \prime} \kappa^{\prime \prime} a^{2}}{v \beta^{\prime \prime}}\right) E^{\prime \prime} q_{2} I_{7}+\frac{\mu_{e} \eta \varepsilon}{4 \pi \rho_{0} v} p_{2} I_{9}\right] \sigma_{r}} \\
=-\left[\frac{I_{1}}{P_{l}}-\left(\frac{g_{0} \lambda \alpha \kappa a^{2}}{v \beta}\right) I_{2}+\left(\frac{g_{0} \lambda \alpha^{\prime} \kappa^{\prime} a^{2}}{v \beta^{\prime}}\right) I_{4}+\left(\frac{g_{0} \lambda \alpha^{\prime \prime} \kappa^{\prime \prime} a^{2}}{v \beta^{\prime \prime}}\right) I_{6}+\frac{\mu_{e} \eta \varepsilon}{4 \pi \rho_{0} v} I_{8}\right], \\
{\left[\left(\frac{1}{\varepsilon}-\frac{F}{P_{l}}\right) I_{1}-\left(\frac{g_{0} \lambda \alpha \kappa a^{2}}{v \beta}\right) E p_{1} I_{3}-\left(\frac{g_{0} \lambda \alpha^{\prime} \kappa^{\prime} a^{2}}{v \beta^{\prime}}\right) E^{\prime} q_{1} I_{5}\right.} \\
\left.-\left(\frac{g_{0} \lambda \alpha^{\prime \prime} \kappa^{\prime \prime} a^{2}}{v \beta^{\prime \prime}}\right) E^{\prime \prime} q_{2} I_{7}-\frac{\mu_{e} \eta \varepsilon}{4 \pi \rho_{0} v} p_{2} I_{9}\right] \sigma_{i}=0 .
\end{gathered}
$$

The integrals $I_{1}, I_{2}, \ldots, I_{9}$ are all positive definite, where

$$
I_{1}=\int_{0}^{1}\left(|D W|^{2}+a^{2}|W|^{2}\right) d z, \quad I_{2}=\int_{0}^{1}\left(|D \Theta|^{2}+a^{2}|\Theta|^{2}\right) d z, \quad I_{3}=\int_{0}^{1}|\Theta|^{2} d z,
$$




$$
\begin{gathered}
I_{4}=\int_{0}^{1}\left(\left.D \Gamma\right|^{2}+a^{2}|\Gamma|^{2}\right) d z, \quad I_{5}=\int_{0}^{1}|\Gamma|^{2} d z, \quad I_{6}=\int_{0}^{1}\left(|D \Psi|^{2}+a^{2}|\Psi| d z, \quad I_{7}=\int_{0}^{1}|\Psi|^{2} d z,\right. \\
I_{8}=\int_{0}^{1}\left(\left|D^{2} K\right|^{2}+2 a^{2}|D K|^{2}+a^{4}|K|^{2}\right) d z, \quad I_{9}=\int_{0}^{1}\left(|D K|^{2}+a^{2}|K| d z .\right.
\end{gathered}
$$

It follows from equation (31) that $\sigma_{r}$ may be positive or negative which means that the system may be stable or unstable. It is clear from (32) that $\sigma_{i}$ may be zero or nonzero, meaning that the modes may be non-oscillatory or oscillatory. The oscillatory modes are introduced due to the presence of kinematic viscoelasticity, varying gravity field, magnetic field and stable solute gradients which were non-existent in their absence.

\section{THE CASE OF OVERSTABILITY}

Here we discuss the possibility of whether instability may occur as overstability. Since we wish to determine the Rayleigh number for the onset of instability via a state of pure oscillations, it suffices to find conditions for which (26) will admit of solutions with $\sigma_{1}$ real.

If we equate real and imaginary parts of (26) and eliminate $R_{1}$ between them, we obtain

$$
A_{3} c_{1}^{3}+A_{2} c_{1}^{2}+A_{1} c_{1}+A_{0}=0,
$$

where we have put $c_{1}=\sigma_{1}^{2}, b=1+x$ and

$$
\begin{gathered}
A_{3}=b\left(E^{\prime} q_{1} E^{\prime \prime} q_{2}\right)^{2}\left(\frac{1}{\varepsilon}-\frac{F}{P}\right)^{2}\left[\frac{E p_{1}}{P}+b\left(\frac{1}{\varepsilon}-\frac{F}{P}\right)\right], \\
A_{0}=b^{5}\left[\frac{b}{P^{2}}\left(\frac{1}{\varepsilon}-\frac{F}{P}\right)+\frac{E p_{1}}{P^{3}}\right]+\frac{b^{3}}{P^{2}}(b-1) \\
\left.\left[S_{1} \lambda\left(E p_{1}-E^{\prime} q_{1}\right)+S_{2} \lambda E p_{1}-E^{\prime \prime} q_{2}\right]\right)^{2}+\frac{b^{3}}{P^{2}} Q_{1}\left(E p_{1}-p_{2}\right) .
\end{gathered}
$$

As $\sigma_{1}$ is real for instability, the four values of $c_{1}\left(=\sigma_{1}^{2}\right)$ must be positive. The product of the roots of (33) is $-A_{0} / A_{3}$, which is possible if

$$
E p_{1}>E^{\prime} q_{1}, E p_{1}>E^{\prime \prime} q_{2}, E p_{1}>p_{2}, \lambda>0 \text { and } \frac{F}{P}<\frac{1}{\varepsilon}
$$


which implies that

$$
E^{\prime} \kappa<E \kappa^{\prime}, \quad E^{\prime \prime} \kappa<E \kappa^{\prime \prime}, \quad \kappa<E \eta, \lambda>0 \text { and } v<\frac{k_{1}}{\varepsilon} .
$$

Equations (37) are, therefore, the necessary conditions for the non-existence of overstability, the violation of which does not necessarily imply the occurrence of overstability.

\section{REFERENCES}

[1] Chandrasekhar S., Hydrodynamic and Hydromagnetic Stability, Dover Publications, New York, 1981.

[2] Kango S.K., Singh V., Thermosolutal convection in Rivlin-Ericksen fluid in the presence of Hall currents in porous medium in hydromagnetics, Int. J. of Applied Mechanics and Engineering, 2012, 17 (1), 71-82.

[3] Nield D.A., Bejan A., Convection in porous medium, Springer, New York, 1992

[4] Oldenburg C., Pruess K., Layered thermohaline convection in hypersaline geothermal systems, Transport in Porous Media, 1998, 33, 29-63.

[5] Pearlstein A.J., Harris R.M., Terrones G., The onset of convective instability in triply diffusive fluid layer, J. Fluid Mech., 1989, 202, 443-465.

[6] Pradhan G.K., Samal P.C., Tripathi U.K., Thermal instability of the fluid layer under variable gravitational field, J. Math., 1989, 20, 736.

[7] Sharma R.C., Sunil, Suresh Chand, Thermosolutal convection in Walters' (Model B') fluid in porous medium in hydrodynamics, Studia Geotechnia et Mechanica, 2000, 3-4, 3-14.

[8] Spiegel E.A., Convective instability in a compressible atmosphere, Astrophys. J., 1965, 141, 1068.

[9] Straughan B.D., Walker W., Multi component diffusion and penetrative convection, Fluid Dynamics Research, 1997, 19, 77-89.

[10] SURESH CHAND, Effect of rotation on triple-diffusive convection in Walters' (Model B) fluid in porous medium, Research J. Science and Tech., 5(1), Jan.-Mar. 2013, 184-188.

[11] Veronis G., Thermohaline convection in a layer of fluid heated from below, J. Marine Res., 1965, 23, 1-17.

[12] Walters K., The motion of an elastico-viscous liquid contained between coaxial cylinders, Quart. J. Mech. Appl. Math., 1960, 13, 444. 